Article

\title{
Microwave Assisted Synthesis of 3, 5-Disubstituted 1, 2, 4-Tria- zole Based Piperazine Amide and Urea Derivatives
}

\author{
Dhanamoorthy Vaithiyalingam, Malini Nelson, Muniyappan Chinnamadhaiyan, Siva Ayyanar*
}

\author{
Supramolecular and Organometallic Chemistry Laboratory, Department of Inorganic Chemistry, School of \\ Chemistry, Madurai Kamaraj University, Madurai-625 021, Tamilnadu, India. \\ *Correspondence: drasiva@gmail.com
}

Abstract: We reported here the microwave assisted synthesis of 3,5-disubstituted 1,2,4-triazole based piperazine amide and urea derivatives with very good yield under mild reaction conditions.

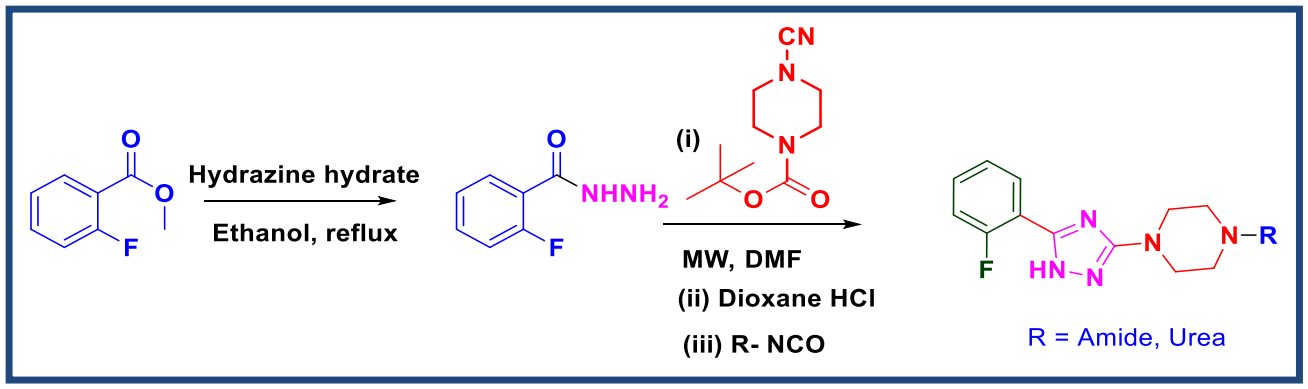

How to cite this paper: Vaithiyalingam, D., Nelson, M., Chinnamadhaiyan, M., \& Ayyanar, S. (2021). Microwave Assisted Synthesis of 3, 5-Disubstituted 1, 2, 4-Triazole Based Piperazine Amide and Urea Derivatives. Online Journal of Chemistry, 1(1), 7-17. DOI:

10.31586/ojc.2021.010102. Retrieved from https://www.scipublications.com/journal/index.php/ojc/article/view/39

Received: June 2, 2021

Accepted: July 16, 2021

Published: July 17, 2021

Copyright: (c) 2021 by the authors. Submitted for possible open access publication under the terms and conditions of the Creative Commons Attribution (CC BY) license (http://creativecommons.org/licenses /by/4.0/).
Keywords: Triazole, piperazine, urea, microwave

\section{Introduction}

Substituted 1,2,4-triazole and its derivatives have gained a considerable interest of the medicinal chemist in search for the new drugs. Because 1,2,4-triazoles are key skeletons of many biologically active molecules and exhibits versatile applications like pesticides, medicines, organocatalysts and functional materials [1,2]. Based on the high importance of this scaffold, the development of methods for its synthesis has been a focus in organic chemistry [3]. Previously, 1,2,4-triazole derivatives were prepared from the $N$ acyl amidrazones obtained from hydrazines and carboxylic acid derivatives by intramolecular cyclization [4]. However, the major drawback of this method is tedious synthetic procedures and low yields. Therefore, the development of a simple and efficient procedure to prepare 1,2,4-triazole derivatives is still essential. Recently, the transition-metalcatalyzed synthesis of 1,2,4-triazoles from readily available starting materials were reported with low yield and harsh conditions [5]. Subsequently, Yeung synthesized 1,2,4triazoles using a convenient and efficient one-pot synthesis of by condensation of nitriles and hydrazides in presence of base [6]. In 2009, Nagasawa's group reported copper-catalyzed synthesis of 1,2,4-triazole derivatives via coupling of amidines with nitriles [7], which was the first example of the synthesis of 1,2,4-triazole derivatives using transitionmetal. In 2014, Huang group report the synthesis of trisubstituted 1,2,4-triazole by a new 1,3-dipolar cycloaddition between oxime and hydrazonoyl chloride under basic condition [8]. In the same year, Beifuss's group reported the synthesis of symmetrically substituted 3,5-diaryl-1,2,4-triazoles using a novel and efficient copper-catalyzed reaction between imidates and ammonium carbonate [9]. A more recent report has shown that nitriles and 
hydroxylamine can also form 1,2,4-triazoles [10]. Kalita et al., have developed iodine mediated metal free synthesis of symmetrical and unsymmetrical 3,5-disubstituted-1H-1,2,4triazole derivatives from amidines and imidates [11].

Here, we report the direct reaction between piperazine substituted nitrile and hydrazide under microwave condition for the efficient synthesis of the intermediate (3,5-substituted 1,2,4-triazoles) with good yield and very less reaction time (Scheme 1). Further, we synthesized a series of various urea derivatives with the use of this intermediate.

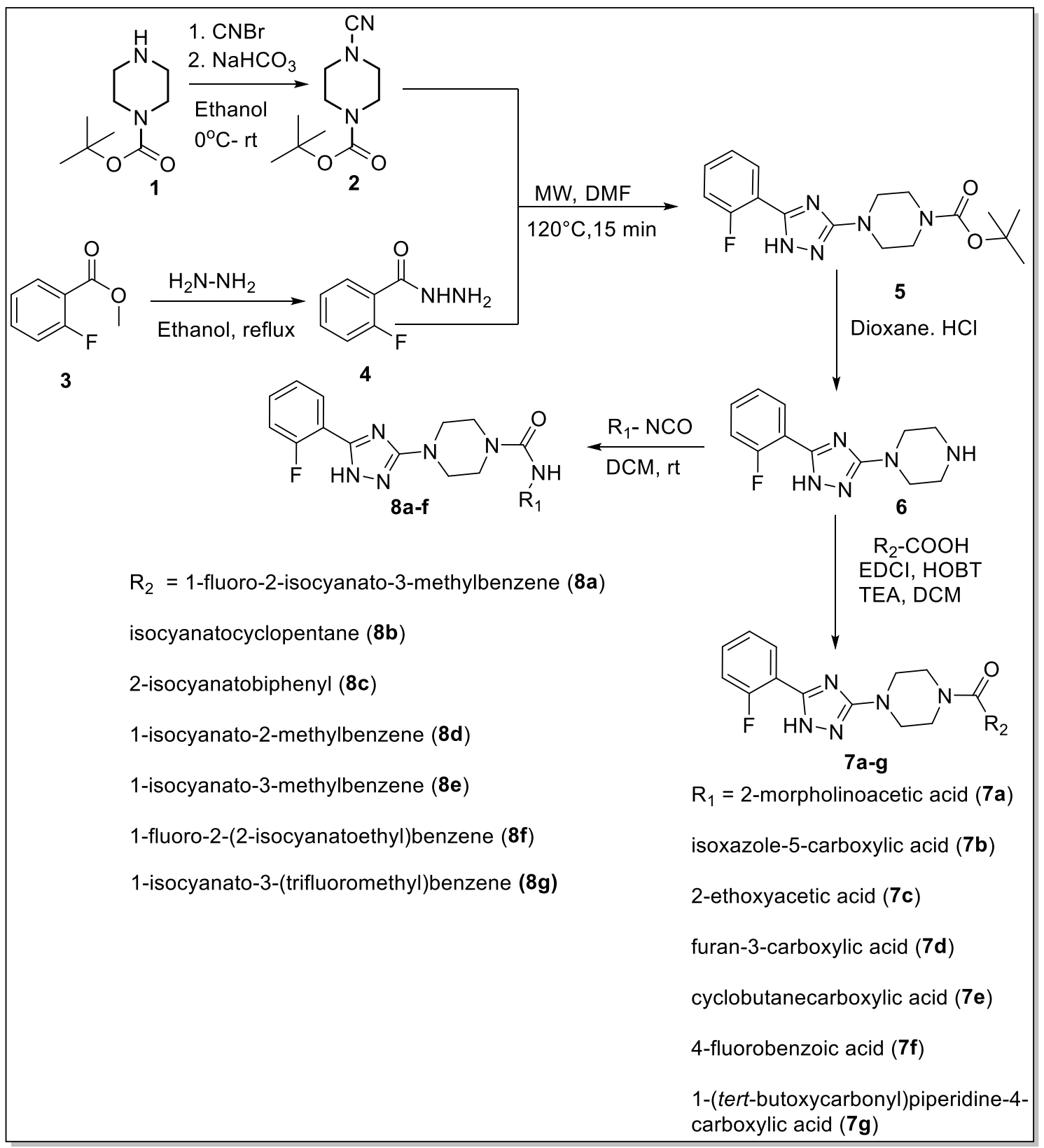

Scheme 1. Synthetic route of 3,5-substituted 1,2,4- triazole based urea and amide derivatives.

\section{Experimental section}

\subsection{Materials and methods}

All the chemicals and reagents were used in this work as an analytical grade. N- BOC piperazine, cyanogen bromide, 2-fluoro methyl benzoate,2-isocyanato 1,1-biphenyl, 2- 
fluoro isocyanate, 3-methyl isocyanate, 3-methyl isocyanate, 1-fluoro-2-isocyanato- 3 propyl benzene, 4-chloro phenyl isocynate, 2-chloro phenyl isocyanate and 4-methoxy phenyl isocyanate were purchased from Sigma Aldrich. Isocyanatocyclopentane, cyclohexyl isocyanate 1-Naphthyl isocyanate, 3- trifluoromethyl phenyl isocyanate were purchased from Alfa Aesar and copper sulphate, hydrazine hydrate and sodium bicarbonate were obtained from Merck and all the solvents were obtained from laboratory grade.

The melting points were measured in open capillary tubes and are uncorrected. The ${ }^{1} \mathrm{H}$ and ${ }^{13} \mathrm{C}$ NMR spectra were recorded on a Bruker (Avance) $300 \mathrm{MHz}$ NMR instrument using TMS as an internal standard, $\mathrm{CDCl}_{3}$ and DMSO-d 6 as a solvent. Standard Bruker software was used throughout. Chemical shifts are given in parts per million ( $\delta$-scale) and the coupling constants are given in Hertz. Silica gel-G plates (Merck) were used for TLC analysis with a mixture of n-hexane and ethyl acetate as an eluent.

\subsection{Preparation of tert butyl 1-cyano piperazine carboxylate (2)}

To a stirred solution of N-bocpiperazine $(9 \mathrm{~g}, 0.048 \mathrm{~mol})$ and $\mathrm{NaHCO}_{3}(4.05 \mathrm{~g}, 0.048$ $\mathrm{mol})$ in ethanol $(60 \mathrm{~mL})$, was added cyanogen bromide $(6.10 \mathrm{~g}$, $0.0576 \mathrm{~mol})$ portion wise at $0^{\circ} \mathrm{C}$. The reaction mixture was stirred at room temperature for about $4 \mathrm{~h}$. After completion of reaction, ethanol was concentrated under reduced pressure, water was added into the residue $(40 \mathrm{~mL})$, extracted with ethyl acetate $(2 \times 40 \mathrm{~mL})$, washed with brine (20 $\mathrm{mL}$ ) dried over anhydrous $\mathrm{Na}_{2} \mathrm{SO}_{4}$ and concentrated under reduced pressure. Yield: $9.2 \mathrm{~g}$ (90.7\%) white solid; m.p. $119-122^{\circ} \mathrm{C}$; $\mathrm{R}_{\mathrm{f}}=0.60$ (UV active, $20 \%$ EtOAc in hexane); ${ }^{1} \mathrm{H}-\mathrm{NMR}$ $\left(300 \mathrm{MHz}, \mathrm{CDCl}_{3}\right) \delta=3.54-3.51(\mathrm{t}, 4 \mathrm{H}), 1.46-1.46(\mathrm{~m}, 9 \mathrm{H}) ; \mathrm{MS}: \mathrm{m} / \mathrm{z}[\mathrm{M}+] 212.30$

\subsection{Preparation of 2-fluorobenzohydrazide (4)}

To a stirred solution of methyl 2-fluorobenzoate $3(6 \mathrm{~g}, 0.038 \mathrm{~mol})$ in ethanol, was added hydrazine hydrate $(2.87 \mathrm{~g}, 0.057 \mathrm{~mol})$ drop wise and gradually heated to $90^{\circ} \mathrm{C}$ and maintained for $5 \mathrm{~h}$. After completion of reaction, the solvent was removed under reduced pressure. The solid was formed, filtered and washed with minimum amount of water.

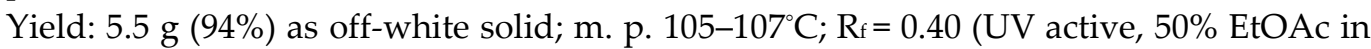
hexane); ${ }^{1} \mathrm{H}$ NMR (300 MHz, DMSO-d 6$) \delta=9.51(\mathrm{~s}, 1 \mathrm{H}), 7.56-7.47(\mathrm{~m}, 2 \mathrm{H}), 7.29-7.22(\mathrm{~m}$, 2H), 4.52 (s, 2H); IR (film) v = 3305, 3198, 1646, $694 \mathrm{~cm}^{-1}$; MS: m/z [M+] 155.30.

\subsection{Preparation of intermediate (5)}

The mixture of 2-fluoro benzohydrazide $4(2.5 \mathrm{~g}, 0.016 \mathrm{~mol})$ and compound $2(3.77 \mathrm{~g}$, $0.017 \mathrm{~mol}$ ) were dissolved in DMF and kept in a microwave at $120^{\circ} \mathrm{C}$ for about 15 mins. After completion of reaction, reaction mixture was concentrated under reduced pressure to remove DMF and added water, extracted with ethyl acetate $(40 \mathrm{~mL})$, washed with brine solution and dried over anhydrous $\mathrm{Na}_{2} \mathrm{SO}_{4}$ and concentrated under reduced pressure. Then the compound was purified by silica gel (60 -120 mesh) using pet ether and ethyl acetate as eluent. Yield: $5.1 \mathrm{~g}\left(92.0 \%\right.$ ) off white solid; $\mathrm{m}$. p. $118-119^{\circ} \mathrm{C} ; \mathrm{R}_{\mathrm{f}}=0.40$ (UV active, $20 \%$ EtOAc in hexane); ${ }^{1} \mathrm{H}-\mathrm{NMR}\left(300 \mathrm{MHz}, \mathrm{MeOD}-\mathrm{d}_{4}\right) \delta=7.95-7.91(\mathrm{~d}, 1 \mathrm{H}), 7.49-7.46(\mathrm{~d}$, 1H),7.31-7.22 (m, 2H), 3.45-3.43 (m, 4H), 2.96-2.94 (m, 4H),1.41 (s, 9H); MS: m/z [M+] 348.00

\subsection{Preparation of 4-\{5-[2-fluorophenyl]-1H-1,2,4-triazol- 3-yl\}piperazine (6)}

To a stirred solution of intermediate $5(4.0 \mathrm{~g}, 0.011 \mathrm{~mol})$ in dioxane $(30 \mathrm{~mL})$, was added dioxane. $\mathrm{HCl}$ at $0^{\circ} \mathrm{C}$ and Stirred for $30 \mathrm{mins}$ at room temperature. After completion of the reaction, the reaction mass was concentrated under reduced pressure. The salt was basified to $\mathrm{PH}^{\mathrm{H}}=9$ by adding $25 \mathrm{~mL}$ of water and $10 \% \mathrm{NaHCO}_{3}$ Solution and extracted with ethyl acetate $(2 \times 25 \mathrm{~mL})$, dried over anhydrous $\mathrm{Na}_{2} \mathrm{SO}_{4}$ and concentrated under reduced pressure. The crude was purified by column chromatography using silica gel (60120 mesh size) using pet ether and ethyl acetate as eluent. Yield: 2.5 g. (89.0\%), off white solid; m. p. $120-122{ }^{\circ} \mathrm{C} ; \mathrm{R}_{\mathrm{f}}=0.20$ (UV active, 50\% EtOAc in hexane); ${ }^{1} \mathrm{H}-\mathrm{NMR}$ (300 MHz, 
MeOD-d 4$) \delta=7.95-7.91(\mathrm{~d}, 1 \mathrm{H}), 7.49-7.46(\mathrm{~d}, 1 \mathrm{H}), 7.31-7.22(\mathrm{~m}, 2 \mathrm{H}), 3.45-3.43(\mathrm{~m}, 4 \mathrm{H}), 2.96-$ 2.94 (m, 4H); IR (film) v = 3277, 3207, 3126, 2952, 2837, 1587, 1520, $767 \mathrm{~cm}^{-1}$; MS: m/z [M+] 248.00 .

\subsection{Preparation of amide derivatives (7a-g) \\ General procedure}

To the well stirred mixture of corresponding carboxylic acid (1.1 eq), EDCI.HCl (1.2 eq) and HOBT (1.2 eq) in DCM, were added triethylamine followed by addition of intermediate 6 at $0^{\circ} \mathrm{C}$ and the reaction mixture was stirred for room temperature for $3 \mathrm{~h}$. After completion of reaction, water was added to reaction mixture. The solid formed was filtered, washed with water, and dried under reduced pressure. The crude was purified by column chromatography using silica gel 230-400 mesh to get compounds 7a-g.

\subsection{1-(4-(5-(2-Fluorophenyl)-1H-1,2,4-triazol-3-yl)piperazin-1-yl)-2- morpholinoethanone (7a)}

Off white solid; $\mathrm{R}_{\mathrm{f}}=0.50$ (UV active, $10 \% \mathrm{CHCl}_{3}$ in $\left.\mathrm{MeOH}\right) ;{ }^{1} \mathrm{H}-\mathrm{NMR}\left(300 \mathrm{MHz}, \mathrm{CDCl}_{3}\right)$ $\delta=10.79(\mathrm{~s}, 1 \mathrm{H}), 8.22-8.18(\mathrm{~m}, 1 \mathrm{H})$, 7.46-7.42 $(\mathrm{m}, 1 \mathrm{H})$, 7.33-7.73 $(\mathrm{m}, 1 \mathrm{H})$, 7.29-7.19 $(\mathrm{m}, 1 \mathrm{H})$, 3.77-3.73 (m, 8H) , 3.57-3.50 (m, 4H), $3.25(\mathrm{~s}, 2 \mathrm{H}), 2.56-2.55(\mathrm{~m}, 4 \mathrm{H}) ;{ }^{13} \mathrm{C}-\mathrm{NMR}(75 \mathrm{MHz}$, $\left.\mathrm{CDCl}_{3}\right) \delta=167.8,161.3,158.9,131.6,131.6,129.8,129.6,129.6,125.0,116.0,115.8,115.3,66.8$, 61.6, 53.4, 47.1, 46.6, 45.1, 41.3; IR(film) $v=3431,3202,2919,2852,1631,1531,1480,755 \mathrm{~cm}$ 1; MS: $\mathrm{m} / \mathrm{z}[\mathrm{M}+]: 375.20$.

\section{8. (4-(5-(2-Fluorophenyl)-1H-1,2,4-triazol-3-yl)piperazin-1-yl)(isoxazol-5- yl)methanone $(7 b)$}

Off white solid; $\mathrm{R}_{\mathrm{f}}=0.45$ (UV active, $10 \% \mathrm{CHCl} 3$ in $\mathrm{MeOH}$ ); ${ }^{1} \mathrm{H}-\mathrm{NMR}(300 \mathrm{MHz}$, $\left.\mathrm{CDCl}_{3}\right) \delta=10.81(\mathrm{~s}, 1 \mathrm{H}), 8.36-8.35(\mathrm{~d}, 1 \mathrm{H}), 8.23-8.19(\mathrm{~m}, 1 \mathrm{H}), 7.46-7.42(\mathrm{~m}, 1 \mathrm{H}), 7.33-7.19$ $(\mathrm{m}, 2 \mathrm{H}), 6.84-6.84(\mathrm{~m}, 1 \mathrm{H}), 3.95-3.88(\mathrm{~m}, 4 \mathrm{H}), 3.67-3.62(\mathrm{~m}, 4 \mathrm{H}) ;{ }^{13} \mathrm{C}-\mathrm{NMR}\left(75 \mathrm{MHz}, \mathrm{CDCl}_{3}\right)$ $\delta=165.2,163.6,161.4,156.9,150.2,131.8,131.7,129.5,125.1,115.6,107.8,47.1,46.4,46.1$, 42.4; IR(film) v = 3099, 2847, 16939, 1593, 1531, 1487, 1421, 1256, $778 \mathrm{~cm}^{-1}$; MS: $\mathrm{m} / \mathrm{z}[\mathrm{M}+]$ : 343.00 .

\subsection{2-Ethoxy-1-(4-(5-(2-fluorophenyl)-1H-1,2,4-triazol-3-yl)piperazin-1-yl)ethanone (7c)}

Off white solid; $\mathrm{R}_{\mathrm{f}}=0.40$ (UV active, $10 \% \mathrm{CHCl}_{3}$ in $\left.\mathrm{MeOH}\right) ;{ }^{1} \mathrm{H}-\mathrm{NMR}\left(300 \mathrm{MHz}, \mathrm{CDCl}_{3}\right)$ $\delta=10.70(\mathrm{~s}, 1 \mathrm{H}), 8.22-8.18(\mathrm{~m}, 1 \mathrm{H}), 7.47-7.41(\mathrm{~m}, 1 \mathrm{H}), 7.32-7.23(\mathrm{~m}, 1 \mathrm{H}), 7.21-7.18(\mathrm{~m}, 1 \mathrm{H})$, $4.21(\mathrm{~s}, 2 \mathrm{H}), 3.78-3.76(\mathrm{~m}, 2 \mathrm{H}), 3.69-3.68(\mathrm{~m}, 2 \mathrm{H}), 3.63-3.59(\mathrm{~m}, 2 \mathrm{H}), 3.57-3.54(\mathrm{~m}, 4 \mathrm{H}), 1.28-$ $1.24(\mathrm{t}, 3 \mathrm{H}) ;{ }^{13} \mathrm{C}-\mathrm{NMR}\left(75 \mathrm{MHz}, \mathrm{CDCl}_{3}\right) \delta=168.1,165.1,161.4,158.9,150.0,131.7,129.6$, 125.0, 115.8, 70.4, 66.8, 46.9, 46.5, 44.7, 41.3, 15.0; IR(film) $v=3093,2978,2850,1654,1594$, 1530, 1493, 779 $\mathrm{cm}^{-1}$; MS: $\mathrm{m} / \mathrm{z}[\mathrm{M}+]: 334.00$.

\subsection{0. (4-(5-(2-Fluorophenyl)-1H-1,2,4-triazol-3-yl)piperazin-1-yl)(furan-3-yl)methanone} (7d)

Off white solid; $\mathrm{R}_{\mathrm{f}}=0.35$ (UV active, $10 \% \mathrm{CHCl}_{3}$ in $\mathrm{MeOH}$ ); ${ }^{1} \mathrm{H}-\mathrm{NMR}\left(300 \mathrm{MHz}, \mathrm{CDCl}_{3}\right.$ ) $\delta=10.68(\mathrm{~s}, 1 \mathrm{H}), 8.22-8.18(\mathrm{~m}, 1 \mathrm{H}), 7.75-7.75(\mathrm{~m}, 1 \mathrm{H}), 7.47-7.42(\mathrm{~m}, 2 \mathrm{H}), 7.33-7.18(\mathrm{~m}, 2 \mathrm{H})$, 6.59-6.59 (m, 1H),3.84-3.75 (m, 4H), 3.60 (m, 4H); ${ }^{13} \mathrm{C}-\mathrm{NMR}\left(75 \mathrm{MHz}, \mathrm{CDCl}_{3}\right) \delta=164.0,161.4$, 158.9, 143.5, 143.0, 131.7, 131.3, 129.6, 125.0, 120.8, 116.0, 115.8, 110.0, 46.9,46.7, 44.6, 42.1; IR(film) $v=3132,2846,1617,1531,1487,1436,751 \mathrm{~cm}^{-1}$; MS: $\mathrm{m} / \mathrm{z} \quad[\mathrm{M}+]: 342.00$.

2.11. cyclobutyl(4-(5-(2-fluorophenyl)-1H-1,2,4-triazol-3-yl)piperazin-1-yl)methanone (7e)

Off white solid; $\mathrm{R}_{\mathrm{f}}=0.35$ (UV active, $10 \% \mathrm{CHCl}_{3}$ in $\mathrm{MeOH}$ ); ${ }^{1} \mathrm{H}-\mathrm{NMR}(300 \mathrm{MHz}$, $\left.\mathrm{CDCl}_{3}\right): \delta=10.68(\mathrm{~s}, 1 \mathrm{H}), 8.22-8.17(\mathrm{~m}, 1 \mathrm{H}), 7.45-7.32(\mathrm{~m}, 1 \mathrm{H}), 7.30-7.23(\mathrm{~m}, 1 \mathrm{H}), 7.21-7.18$ $(\mathrm{m}, 1 \mathrm{H}), 3.78-3.75(\mathrm{~m}, 2 \mathrm{H}), 3.52-3.49(\mathrm{~m}, 6 \mathrm{H}), 3.34-3.30(\mathrm{~m}, 1 \mathrm{H}), 2.42-2.37(\mathrm{~m}, 2 \mathrm{H}), 2.21-2.12$ $(\mathrm{m}, 2 \mathrm{H}), 2.00-1.90(\mathrm{~m}, 2 \mathrm{H}) ;{ }^{13} \mathrm{C}-\mathrm{NMR}\left(75 \mathrm{MHz}, \mathrm{CDCl}_{3}\right) \delta=173.3,164.7,161.3,158.3,150.12$, 
131.6, 129.6, 124.9, 115.7, 46.8, 46.5, 44.4, 41.0, 37.1, 25.0, 24.9, 17.8;IR(film) v = 3195, 2940, 2860, 1622, 1531, 1438, 1221, $754 \mathrm{~cm}^{-1}$; MS: m/z [M+]: 330.10.

\subsection{2. (4-Fluorophenyl)(4-(5-(2-fluorophenyl)-1H-1,2,4-triazol-3-yl)piperazin-1-} yl)methanone (7f)

Off white solid; $\mathrm{R}_{\mathrm{f}}=0.50$ (UV active, $10 \% \mathrm{CHCl}_{3}$ in $\left.\mathrm{MeOH}\right) ;{ }^{1} \mathrm{H}-\mathrm{NMR}(300 \mathrm{MHz}$, $\left.\mathrm{CDCl}_{3}\right): \delta=10.62(\mathrm{~s}, 1 \mathrm{H}), 8.22-8.17(\mathrm{~m}, 1 \mathrm{H}), 7.49-7.42(\mathrm{~m}, 4 \mathrm{H}), 7.32-7.27(\mathrm{~m}, 1 \mathrm{H}), 7.24-7.10$ $(\mathrm{m}, 2 \mathrm{H}), 3.91-3.58(\mathrm{~m}, 8 \mathrm{H}) ;{ }^{13} \mathrm{C}$ NMR $\left(75 \mathrm{MHz}, \mathrm{CDCl}_{3}\right) \delta=169.7,165.1,164.7,162.2,161.4$, $158.9,150.1,131.7,131.5,129.6,129.4,125.1,116.0,115.8,115.1,115.0,46.7,46.5,116.0,115.8$, 115.1, 46.7, 46.5, 44.3, 41.3; IR(film) $v=3423,3206,2922,2856,1611,1532,1566,756 \mathrm{~cm}^{-1}$; MS: $\mathrm{m} / \mathrm{z}[\mathrm{M}+]: 370.00$.

\subsection{Tert-butyl 4-(4-(5-(2-fluorophenyl)-1H-1,2,4-triazol-3-yl)piperazine-1- carbonyl)piperidine-1-carboxylate (7g)}

Off white solid; $\mathrm{R}_{\mathrm{f}}=0.40$ (UV active, $10 \% \mathrm{CHCl}_{3}$ in $\left.\mathrm{MeOH}\right) ;{ }^{1} \mathrm{H}-\mathrm{NMR}(300 \mathrm{MHz}$, $\left.\mathrm{CDCl}_{3}\right): \delta=10.62(\mathrm{~s}, 1 \mathrm{H}), 8.22-8.18(\mathrm{~m}, 1 \mathrm{H}), 7.45-7.43(\mathrm{~m}, 1 \mathrm{H}), 7.33-7.27(\mathrm{~m}, 1 \mathrm{H}), 7.23-7.18$ $(\mathrm{m}, 1 \mathrm{H}), 4.19-4.12(\mathrm{~m}, 1 \mathrm{H}), 3.78-3.66(\mathrm{~m}, 4 \mathrm{H}), 3.55-3.52(\mathrm{~m}, 4 \mathrm{H}), 2.81-2.69(\mathrm{~m}, 4 \mathrm{H}), 1.79-1.73$ $(\mathrm{m}, 4 \mathrm{H}), 1.47(\mathrm{~s}, 9 \mathrm{H}) ;{ }^{13} \mathrm{C}-\mathrm{NMR}\left(75 \mathrm{MHz}, \mathrm{CDCl}_{3}\right) \delta=173.1,165.0,161.4,158.9,154.7,150.0$, 131.8, 129.6, 125.1, 125.0, 116.0, 115.1, 79.6, 60.3, 47.1, 46.6, 44.9, 43.4, 41.2, 38.4, 28.4, 21.0, 14.1; IR(film) $v=3453,3095,2975,2856,1736,1692,1635,1532,755 \mathrm{~cm}^{-1} ; \mathrm{MS}: \mathrm{m} / \mathrm{z}$ [M+]: 459.00 .

\subsection{Preparation of urea derivatives (8a-g)}

\section{General procedure}

To a well stirred mixture of corresponding intermediate 6 (1.0 eq) and triethylamine (2.0 eq) in DCM were added various isocyanate $(1.1 \mathrm{eq})$ at $0^{\circ} \mathrm{C}$ and the reaction mixture was stirred for room temperature for about $3 \mathrm{~h}$. After completion of reaction, the reaction mixture was concentrated under reduced pressure to remove DCM. The crude product was purified by column chromatography using silica gel 230-400 mesh to get compounds 8a-g.

2.15. $N$-(2-fluoro-6-methylphenyl)-4-(5-(2-fluorophenyl)-1H-1,2,4-triazol-3yl)piperazine-1-carboxamide (8a)

Off white solid; $\mathrm{R}_{\mathrm{f}}=0.45$ (UV active, $10 \% \mathrm{CHCl}_{3}$ in $\left.\mathrm{MeOH}\right) ;{ }^{1} \mathrm{H}-\mathrm{NMR}(300 \mathrm{MHz}$, DMSO-d 6$) \delta=13.12(\mathrm{~s}, 1 \mathrm{H}), 8.17(\mathrm{~s}, 1 \mathrm{H}), 7.95-7.91(\mathrm{~m}, 1 \mathrm{H}), 7.45(\mathrm{~s}, 1 \mathrm{H}), 7.34-7.29(\mathrm{~m}, 2 \mathrm{H})$, 7.21-7.12 (m, 2H), 6.88-6.83 (m, 1H), 3.57-3.41 (m, 4H), 3.36-3.25 (m, 4H), $2.23(\mathrm{~s}, 3 \mathrm{H})$; IR (film) $v=3251,3078,2923,2855,1743,1635,1598,1523,753 \mathrm{~cm}^{-1} \mathrm{MS}: \mathrm{m} / \mathrm{z}$ [M+]: 399.20 .

2.16. $N$-cyclopentyl-4-(5-(2-fluorophenyl)-1H-1,2,4-triazol-3-yl)piperazine-1carboxamide $(8 b)$

Off white solid; $\mathrm{R}_{\mathrm{f}}=0.45$ (UV active, $10 \% \mathrm{CHCl}_{3}$ in $\left.\mathrm{MeOH}\right) ;{ }^{1} \mathrm{H}-\mathrm{NMR}(300 \mathrm{MHz}$, DMSO-d $) \delta=12.83(\mathrm{~s}, 1 \mathrm{H}), 7.92(\mathrm{~s}, 1 \mathrm{H}), 7.26-7.25(\mathrm{~m}, 3 \mathrm{H}), 6.35(\mathrm{~s}, 1 \mathrm{H}), 3.94-3.88(\mathrm{~m}, 4 \mathrm{H})$, 3.40-3.35 (m, 4H), 1.80-1.74 (m, 2H), 1.64-1.59 (m, 2H), 1.49-1.40 (m, 4H); IR (film) v = 3284, 2957, 2863, 1621, 1529, 1483, 1255 $\mathrm{cm}^{-1} \mathrm{MS}: \mathrm{m} / \mathrm{z}[\mathrm{M}+]: 359.30$.

\subsection{7. $N$-(biphenyl-2-yl)-4-(5-(2-fluorophenyl)-1H-1,2,4-triazol-3-yl)piperazine-1-} carboxamide (8c)

Off white solid; $\mathrm{R}_{\mathrm{f}}=0.45$ (UV active, $10 \% \mathrm{CHCl}_{3}$ in $\left.\mathrm{MeOH}\right) ;{ }^{1} \mathrm{H}-\mathrm{NMR}(300 \mathrm{MHz}$, DMSO-d $) ~ \delta=12.83(\mathrm{~s}, 1 \mathrm{H}), 8.11-8.06(\mathrm{~d}, 1 \mathrm{H}), 7.93-7.91(\mathrm{~d}, 1 \mathrm{H}), 7.51(\mathrm{~m}, 1 \mathrm{H}), 7.38-7.28(\mathrm{~m}$, $1 \mathrm{H})$, 3.39-3.32 (m, 4H), 3.32-3.29 (m, 4H); MS: m/z [M+]: 443.30 .

\subsection{4-(5-(2-Fluorophenyl)-1H-1,2,4-triazol-3-yl)-N-m-tolylpiperazine-1-carboxamide} $(8 \mathrm{~d})$

Off white solid; $\mathrm{R}_{\mathrm{f}}=0.45$ (UV active, $10 \% \mathrm{CHCl}_{3}$ in $\left.\mathrm{MeOH}\right) ;{ }^{1} \mathrm{H}-\mathrm{NMR}(300 \mathrm{MHz}$, MeOD): $\delta=7.95$ (s, 1H), 7.52 (s, 1H), 7.3-7.15 (m, 5H), $3.68(\mathrm{~m}, 4 \mathrm{H}), 3.51$ (m, 4H), 2.30 (s, 
3H); IR (film) $v=3267,2914,2831,1639,1537,1484,1451,1300,1248,817 \mathrm{~cm}^{-1} ; \mathrm{MS}: \mathrm{m} / \mathrm{z}$ $[\mathrm{M}+]: 381.40$.

\subsection{4-(5-(2-Fluorophenyl)-1H-1,2,4-triazol-3-yl)- $N$-o-tolylpiperazine-1-carboxamide} (8e)

Off white solid; $\mathrm{R}_{\mathrm{f}}=0.45$ (UV active, $10 \% \mathrm{CHCl}_{3}$ in $\mathrm{MeOH}$ ); ${ }^{1} \mathrm{H}-\mathrm{NMR}(300 \mathrm{MHz}$, DMSO-d 6$) \delta=13.01(\mathrm{~s}, 1 \mathrm{H}), 9.47-9.45(\mathrm{~m}, 1 \mathrm{H}), 8.17-7.96(\mathrm{~d}, 1 \mathrm{H}), 7.96-7.92(\mathrm{~m}, 1 \mathrm{H}), 7.45-7.27$ $(\mathrm{m}, 4 \mathrm{H}), 7.19-7.11(\mathrm{~m}, 1 \mathrm{H}), 7.06-7.02(\mathrm{~m}, 1 \mathrm{H}), 3.59-3.41(\mathrm{~m}, 4 \mathrm{H}), 3.40-3.34(\mathrm{~m}, 4 \mathrm{H}), 2.02(\mathrm{~s}$, 3H); IR (film) $v=3278,2922,2853,1634,1534,1523,1491,1330,1255,994,752 \mathrm{~cm}^{-1} ; \mathrm{MS}: \mathrm{m} / \mathrm{z}$ $[\mathrm{M}+]$ : 381.00 .

\subsection{0. $N$-(2-fluorophenethyl)-4-(5-(2-fluorophenyl)-1H-1,2,4-triazol-3-yl) piperazine-1-} carboxamide (8f)

Off white solid; $\mathrm{R}_{\mathrm{f}}=0.45$ (UV active, $10 \% \mathrm{CHCl}_{3}$ in $\mathrm{MeOH}$ ); ${ }^{1} \mathrm{H}-\mathrm{NMR}(300 \mathrm{MHz}$, DMSO-d 6$): \delta=13.12(\mathrm{~s}, 1 \mathrm{H}), 7.94-7.90 \mathrm{~m}, 1 \mathrm{H}), 7.46-7.25(\mathrm{~m}, 3 \mathrm{H}), 7.24-7.10(\mathrm{~m}, 2 \mathrm{H}), 6.79-$ $6.76(\mathrm{~m}, 1 \mathrm{H}), 3.40-3.24(\mathrm{~m}, 4 \mathrm{H}), 3.22-3.19(\mathrm{~m}, 4 \mathrm{H}), 2.78-2.69(\mathrm{~m}, 2 \mathrm{H}), 2.49-2.49(\mathrm{~m}, 2 \mathrm{H})$; $\operatorname{IR}\left(\right.$ film) $v=3322,2924,2854,1617,1539,1487,1451,1261,1225,754 \mathrm{~cm}^{-1}$; MS: $\mathrm{m} / \mathrm{z}[\mathrm{M}+]$ : 413.00 .

\subsection{4-(5-(2-Fluorophenyl)-1H-1,2,4-triazol-3-yl)- $N$-(3-(trilfuoromethyl)} phenyl)piperazine-1-carboxamide (8g)

Off white solid; $\mathrm{R}_{\mathrm{f}}=0.47$ (UV active, $10 \% \mathrm{CHCl}_{3}$ in $\mathrm{MeOH}$ ); ${ }^{1} \mathrm{H}-\mathrm{NMR}(300 \mathrm{MHz}$, DMSO-d 6$) \delta=13.01(\mathrm{~s}, 1 \mathrm{H}), 9.47-9.45(\mathrm{~m}, 1 \mathrm{H}), 8.17-7.96(\mathrm{~d}, 1 \mathrm{H}), 7.96-7.92(\mathrm{~m}, 1 \mathrm{H}), 7.45-7.27$ $(\mathrm{m}, 4 \mathrm{H}), 7.19-7.11(\mathrm{~m}, 1 \mathrm{H}), 7.06-7.02(\mathrm{~m}, 1 \mathrm{H}), 3.59-3.41(\mathrm{~m}, 4 \mathrm{H}), 3.40-3.34(\mathrm{~m}, 4 \mathrm{H}) ; \quad$ IR (film) $v=3278,2922,2853,1634,1534,1523,1491,1330,1255,994,752 \mathrm{~cm}^{-1}$.

\section{Results and discussion}

Based on above initial information's, we planned to optimize the reaction condition of intermediate 5 . The reaction between nitrile (4) and hydrazide (3) was used to identify the reaction parameters that would provide optimal results. As presented in Table 1, to improve yields and demonstrate the methodology. To this effort, we decided to evaluate the various solvents and bases with microwave heating conditions.

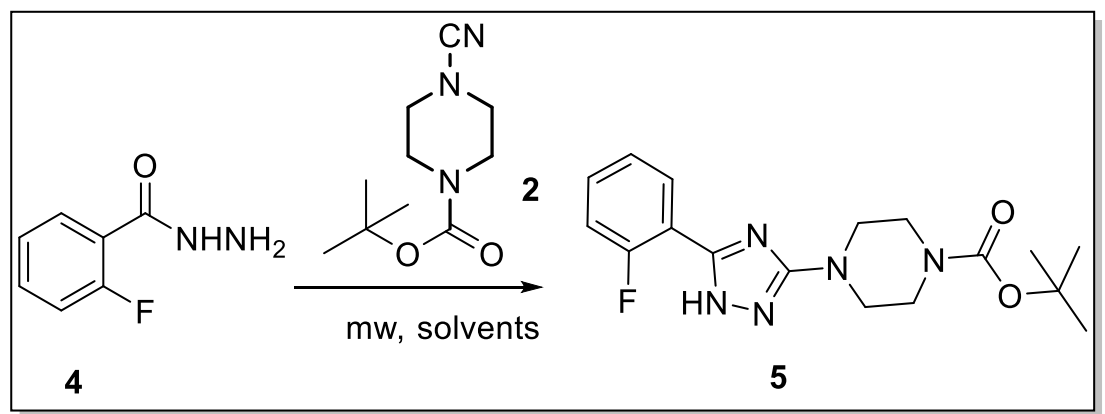

Table 1. Results of the reaction of nitrile with hydrazide under different conditions.

\begin{tabular}{|c|c|c|c|c|}
\hline S. No. & Solvents & Mw $\left({ }^{\circ} \mathrm{C}\right)$ & Time $(\mathrm{mins})$ & Yield $(\%)$ \\
\hline 1. & Ethanol & 90 & 60 & 25 \\
\hline 2. & Butanol & 90 & 50 & 40 \\
\hline 3. & Tert-butanol & 90 & 30 & 50 \\
\hline 4. & Xylene & 120 & 30 & 60 \\
\hline 5. & DMF & $\mathbf{1 2 0}$ & $\mathbf{1 5}$ & $\mathbf{9 2}$ \\
\hline 6. & DMSO & 150 & 20 & 75 \\
\hline
\end{tabular}


Further, we examined different the reaction condition of synthesis of intermediate 5 with convention heating and it compared as shown in Table 2.

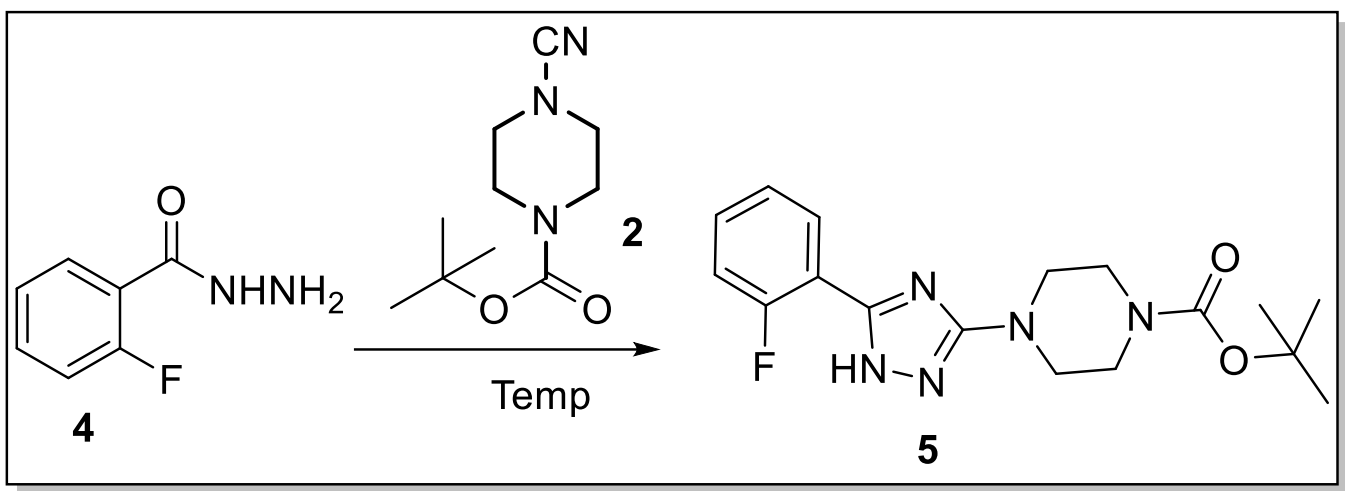

Table 2. Results of the reaction of nitrile with hydrazide under different conditions.

\begin{tabular}{|c|c|c|c|c|c|}
\hline \multirow{2}{*}{ S no } & \multirow{2}{*}{ Solvents } & \multicolumn{2}{|c|}{ Conventional heating } & \multicolumn{2}{|c|}{ MW } \\
\cline { 3 - 6 } & & Temp $\left({ }^{\circ} \mathrm{C}\right)$ & Yield $(\%)$ & Temp $\left({ }^{\circ} \mathrm{C}\right)$ & Yield $(\%)$ \\
\hline 1. & Ethanol & 90 & - & 90 & 20 \\
\hline 2. & Butanol & 90 & 10 & 90 & 40 \\
\hline 3. & Tert-butanol & 90 & 25 & 90 & 50 \\
\hline 4. & Xylene & 120 & 30 & 120 & 60 \\
\hline 5. & DMF & $\mathbf{1 2 0}$ & $\mathbf{5 0}$ & $\mathbf{1 2 0}$ & $\mathbf{9 2}$ \\
\hline 6. & DMSO & 150 & 40 & 150 & 75 \\
\hline
\end{tabular}

Based on the above results, we comparing the conventional heating with microwave condition, MW mediated reactions are the best method for the synthesis of intermediate 5 without using any base or metal. In this method, it takes only few minutes to complete the reaction and it offers good yield with easy work up, when compared to conventional heating method.

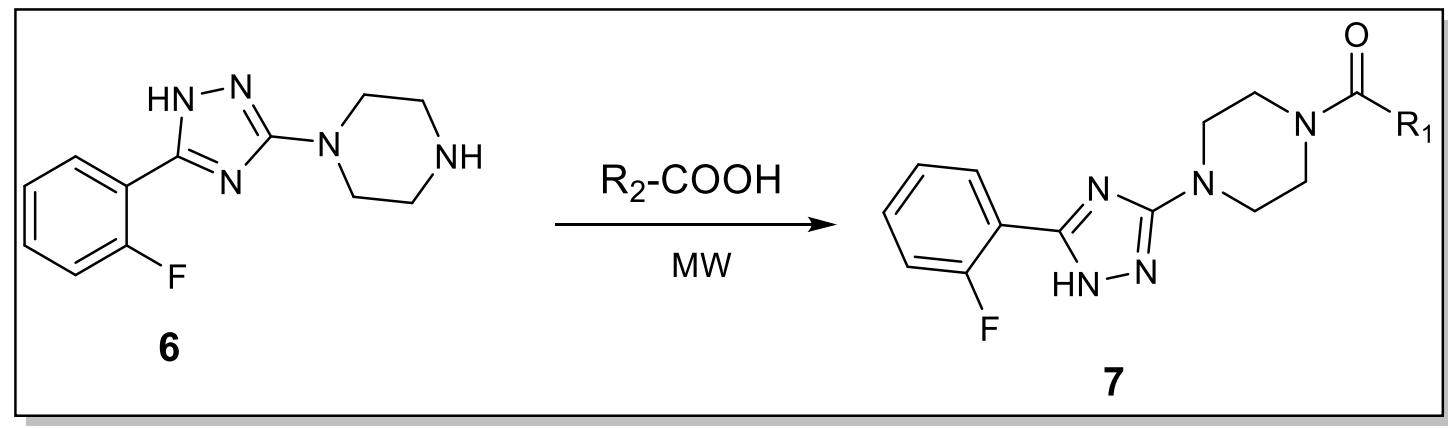


Table 3. Results of the reaction of intermediate 6 with various carboxylic acids.

\begin{tabular}{|c|c|c|c|c|}
\hline $\begin{array}{l}\text { S. } \\
\text { No }\end{array}$ & Acids & Amide derivatives & $\begin{array}{l}\text { Product } \\
\text { code }\end{array}$ & Yield (\%) \\
\hline 1. & & & $7 a$ & 80 \\
\hline 2. & & & $7 \mathrm{~b}$ & 78 \\
\hline 3. & & & $7 c$ & 87 \\
\hline 4. & & & $7 d$ & 85 \\
\hline 5. & & & $7 \mathrm{e}$ & 89 \\
\hline 6. & & & $7 f$ & 90 \\
\hline 7. & & & $7 \mathrm{~g}$ & 88 \\
\hline
\end{tabular}

Further, the intermediate 6 reactions with various isocyanates to give urea derivatives with electron donating and electron withdrawing substituents in the aromatic [1217]. As shown in Table 4, both electron donating and electron withdrawing substituents on phenyl rings does not affect the reaction, and the corresponding reactions proceeded smoothly to afford the desired products with excellent yield in shorter reaction time. 


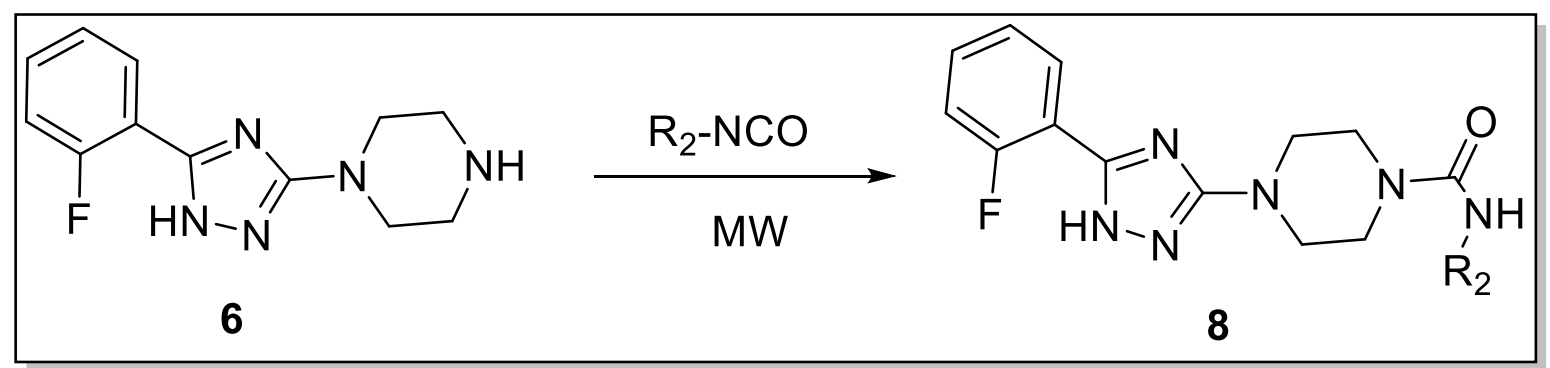

Table 4. Results of the reaction of intermediate 6 with various isocyanates.

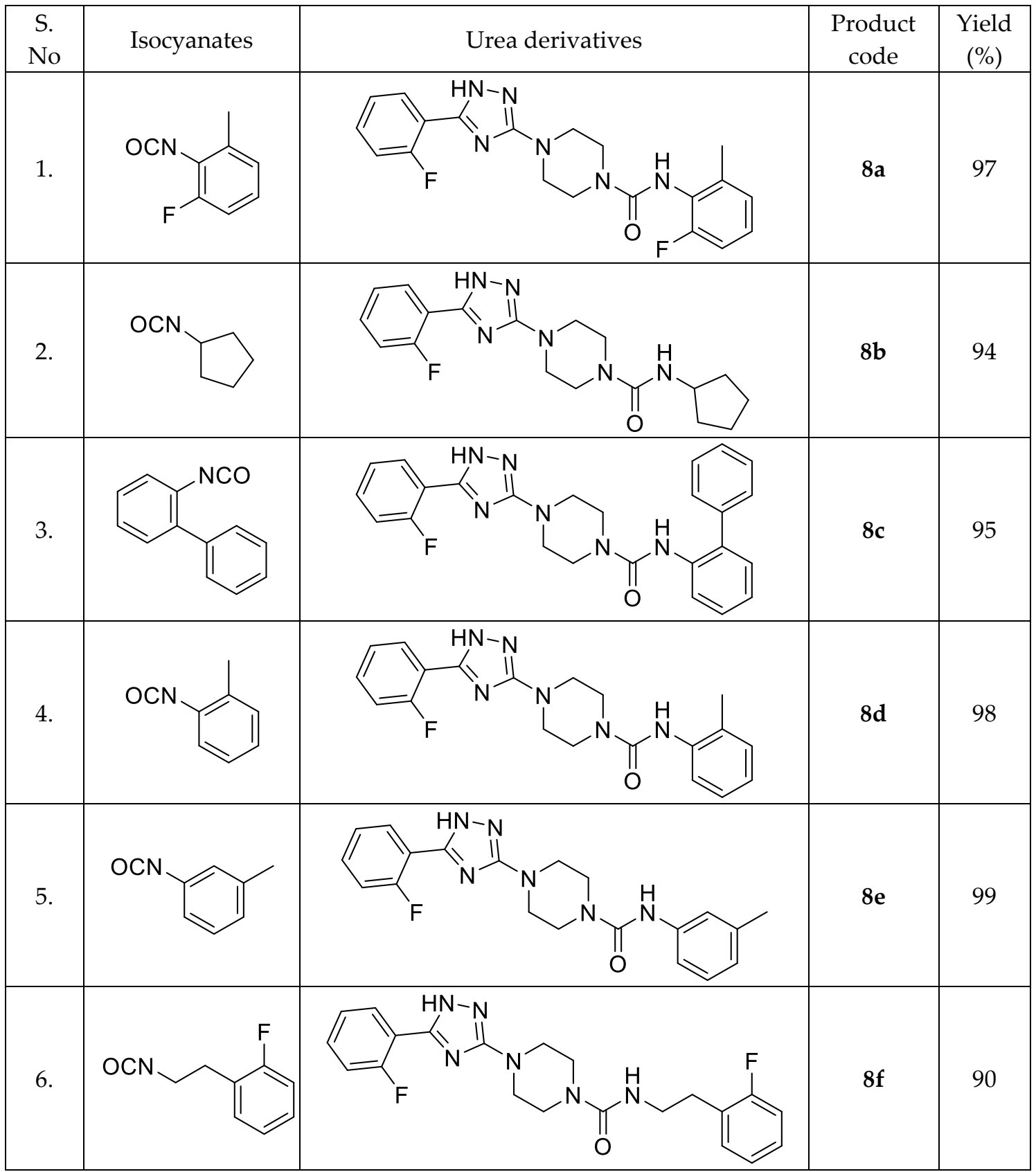




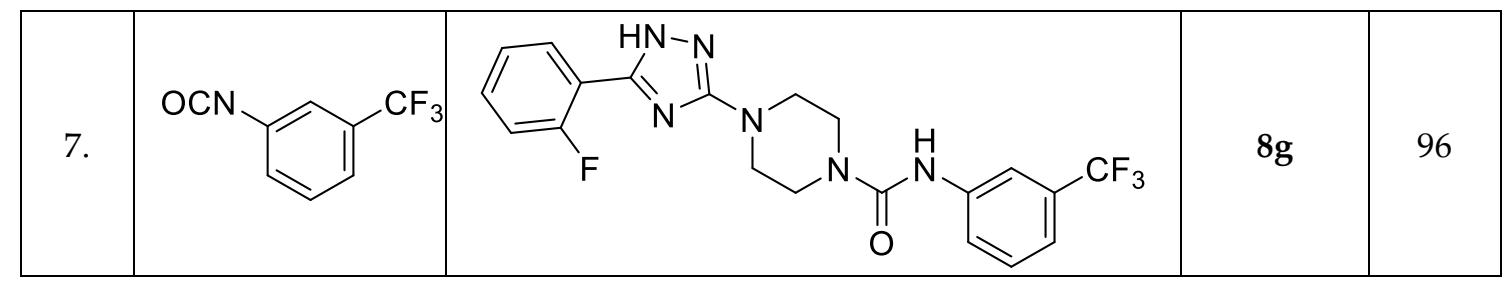

\section{Conclusion}

We synthesized piperazine linked 3,5-disubstituted 1,2,4-triazole intermediate that proceeds by the direct reaction of a nitrile and a hydrazide in microwave irradiation without using any base and solvents. The obtained triazole derivatives as an intermediate for preparing some urea and amide derivatives using their corresponding isocyanates and carboxylic acids through coupling reaction to give very good yield (up to $99 \%$ ) in very short time.

\section{Acknowledgement}

We acknowledge the financial support of the Department of Science and Technology, CERI, (Grant No. DST/TM/CERI/C130(G)), DST-SERB, extramural major research (Grant No. EMR/2015/000969), Council of Scientific and Industrial Research (CSIR) HRDG, New Delhi (Gant No. 01(2901)/17/EMR-II). Also, we thank DST-PURSE and UGC-UPE programs for providing instrumental facility.

\section{References}

[1] a) Tao, Y.; Wang, Q.; Ao, L.; Zhong, C.; Yang, C.; Qin, J.; Ma, D. Highly efficient phosphorescent organic light-emitting diodes hosted by 1,2,4-triazole-cored triphenylamine derivatives: relationship between structure and optoelectronic properties. J. Phys. Chem. C 2010, 114, 601-609. b) Wu, P.L.; Feng, X.J.; Tam, H.L.; Wong, M.S.; Cheah, K.W. Efficient three-photon excited deep blue photoluminescence and lasing of diphenylamino and 1,2,4-triazole endcapped oligofluorenes. J. Am. Chem. Soc. 2009, 131, 886887; c) Nepal, B.; Scheiner, S. Substituent effects on the binding of halides by neutral and dicationic bis(triazolium) receptors. J. Phys. Chem. 2015, 119, 13064-13073.

[2] a) Hull, J.W.; Romer, D.R.; Adaway, T.J.; Podhorez, D.E. Development of manufacturing processes for a new family of 2,6dihaloaryl 1,2,4-triazole insecticides. Org. Process Res. Dev. 2009, 13, 1125-1129; b) Sun, J.; Zhang, A.; Zhang, J.; Xie, X.; Liu, W. Enantiomeric resolution and growth-retardant activity in rice seedlings of uniconazole. J. Agric. Food. Chem. 2012, 60, 160164; c) Al-Masoudi, I.A.; Al-Soud, Y.A.; Al-Salihi, N.J.; Al-Masoudi, N.A. 1,2,4-Triazoles: Synthetic approaches and pharmacological importance. Chem. Heterocycl. Compd. (N.Y.) 2006, 42, 1377-1403.

[3] a) Moulin, A.; Bibian, M.; Blayo, A.L.; Habnouni, S.E.; Martinez, J.; Fehrentz, J.A. Synthesis of 3,4,5-trisubstituted-1,2,4-triazoles. Chem. Rev. 2010, 110, 1809-1827. b) Holm, S.C.; Straub, B.F.; Synthesis of n-substituted 1,2,4-triazoles. a review. Org. Prep. Proced.Int. 2011, 43,319-347.

[4] a) Larsen, S.D.; Di Paolo, B.A. Traceless solid-phase synthesis of 1,2,4-triazoles using a novel amine resin. Org. Lett. 2001, 3, 33413344. b) Stocks, M.J.; Cheshire, D.R.; Reynolds, R. Efficient and regiospecific one-pot synthesis of substituted 1,2,4-triazoles.Org. Lett. 2004, 6, 2969-2971. c) Balsells, J.; DiMichele, L.; Liu, J.; Kubryk, M.; Hansen, K.; Armstrong, J.D. Synthesis of [1,2,4] triazolo[4,3- $\alpha$ ] piperazines via highly reactive chloromethyloxadiazoles. Org. Lett. 2005, 7, 1039-1042.

[5] a) Kearney, A.M.; Vanderwal, C.D. Synthesis of nitrogen heterocycles by the ring opening of pyridinium salts. Angew. Chem., Int. Ed. 2006, 45, 7803-7806. b) Ackermann, L.; Althammer, A. Domino N-H/C-H bond activation: palladium-catalyzed synthesis of annulated heterocycles using dichloro(hetero)arenes. Angew. Chem., Int. Ed. 2007, 46, 1627-1629. c) Seregin, I.V.; Ryabova, V.; Gevorgyan. V. Direct palladium-catalyzed alkynylation of n-fused heterocycles. J. Am. Chem. Soc. 2007, 129, 7742-7743. d) Chuprakov, S.; Gevorgyan. V. Regiodivergent metal-catalyzed rearrangement of 3-iminocyclopropenes into n-fused heterocycles. Org. Lett. 2007, 9, 4463-4466. E) Nagamochi, M.; Fang, Y.Q.; Lautens, M. A general and practical method of alkynyl indole and benzofuran synthesis via tandem cu- and pd-catalyzed cross-couplings. Org. Lett. 2007, 9, 2955-2958.

[6] Yeung, K.S.; Farkas, M.E.; Kadow, J.F.; Meanwell, N.A. A base-catalyzed, direct synthesis of 3,5-disubstituted 1,2,4-triazoles from nitriles and hydrazides. TetrahederonLett., 2005, 46, 3429-3432.

[7] Ueda, S.; Nagasawa, H. Facile synthesis of 1,2,4-triazoles via a copper-catalyzed tandem addition-oxidative cyclization. J. Am. Chem. Soc. 2009, 131, 15080-15081. 
[8] Wang, L.Y.; Tsai, H. J.; Lin, H.Y.; Kaneko, K.; Cheng, F.Y.; Shih, H.S.; Wong, F. F.; Huang, J. J. One-flask synthesis of 1,3,5trisubstituted 1,2,4-triazoles from nitriles and hydrazonoyl chlorides via 1,3-dipolar cycloaddition. RSC Adv., 2014,4, 1421514220.

[9] Sudheendran, K.; Schmidt, D.; Frey, W.; Conrad, J.; Beifuss, U. Facile synthesis of 3,5-diaryl-1,2,4-triazoles via copper-catalyzed domino nucleophilic substitution/oxidative cyclization using amidines or imidates as substrates. Tetrahedron., 2014, 70, 16351645.

[10] Xu, H.; Ma, S.; Xu, Y.; Bian, L.; Ding, T.; Fang, X.; Zhang, W.; Ren, Y. Copper-catalyzed one-pot synthesis of 1,2,4-triazoles from nitriles and hydroxylamine. J. Org. Chem. 2015, 80, 1789-1794.

[11] SurendraBabu, I.; Biswajit, K.; Jafar Ahamed, A. I2 mediated one-pot synthesis of 1,2,4-triazoles from amidines and imidates. Tetrahedron Lett.2016, 57, 2227-2230.

[12] Ayman, E.; Mercedes, A.; Jose, A.E.; Fernando, A. Use of N-Methylpiperazine for the preparation of piperazine-based unsymmetrical bis-ureas as anti-hiv agents. ChemMedChem 2008, 3, 1034-1037.

[13] Cumming, J.G.; MacFaulb, P.; Leach, A.G. Novel N-thiazolyl piperazine-1-carboxamide CCR2 antagonists - investigation of an unexpected reaction with glutathione. Med. Chem. Commun. 2015,6, 2140-2145.

[14] Iriepaa, I.; Bellanatob, J. Synthesis, spectroscopic, structural and conformational study of some tri-substituted ureas derived from N-methylpiperazine containing phenyl and N-heterocyclic substituents. Journal of Molecular Structure, 2013, 1044, 215-220.

[15] John, M.K.; Rich, A.; Tichenor, M.; Xiao, W.; Jones, W.; Pierce, J.; Seierstad, M.; Palmer, J.; Webb, M.; Karbarz, M.; Scott, B.; Wilson, S.; Luo, L.; Wennerholm, M.; Chang, L.; Brown, S.; Rizzolio, M.; Rynberg, R.; Chaplan, S.; Guy Breitenbucher, J. Aryl piperazinyl ureas as inhibitors of fatty acid amide hydrolase (faah) in rat, dog, and primate. ACS Med. Chem. Lett. 2012, 3, 823-827.

[16] Sharma, A.; Suhas, R.; Chandana, K.V.; HajiraBanu, S.; Channe Gowda, D. Tert-Butyl 1,5-bis(4-(benzo[d]isothiazol-3-yl) piperazin-1-yl)-1,5-dioxopentan-2-ylcarbamate urea/thiourea derivatives as potent $\mathrm{H}+/ \mathrm{K}+-\mathrm{ATPase}$ inhibitors. Bioorg. Med. Chem. Lett. 2013, 23, 4096-4098.

[17] Suyoga Vardhan, D.M.; Shantharam, C.S.; Suhas, R.; Channe Gowda, D. Synthesis and evaluation of novel ureido/thioureido derivatives of amino acid conjugated 2,3-dichlorophenyl piperazine as highly potent antiglycating agents. Journal of Saudi Chemical Society., 2017, 21, 248-257. 